\title{
A new multi-robot search algorithm using Probabilistic Finite State Machine and Lennard-Jones potential function
}

\author{
Muhammad S. A. Khan ${ }^{1}$, Mohammad S. Hasan ${ }^{2}$, and Tarem Ahmed ${ }^{3}$
}

\begin{abstract}
Swarm robotics is a decentralized approach to robotic systems. This paper investigates the problem of search and rescue using swarm robots. A multi-robot search algorithm using probabilistic finite state machine and interaction inspired by Lennard-Jones potential function has been proposed. Probabilistic finite state machine has been used to separate the tasks performed and to change coordination rules according to the circumstances and social probabilities. The approach is tested in various scenarios to test flexibility, scalability and robustness. The performance result is promising. Algorithmic complexity comparison with Robotic Darwinian Particle Swarm Optimization and Glowworm Swam Optimization appear favourable.
\end{abstract}

\section{INTRODUCTION}

Within multi-robot systems swarm robotics is a novel approach, taking as inspiration biological swarms like social insects (bees, ants, or termites), fish schools, bird flocks, or bacteria colonies [1]. It is a decentralised approach to robotics that is based on robustness, flexibility, and scalability [2]. From a computation point of view, swarm intelligence simulates the overall behaviour of the swarm and not the individuals in the swarm it is trying to mimic. Specifically, it is the emergent collective behaviour in decentralized groups of autonomous robots with individuals in the swarm following simple local rules which can produce largely varied and complex behaviour for the swarm [1]. The robots themselves are relatively simple in design with limited range of sensors or actuators. The communication between robots is local and limited.

There are several tasks within the field of swarm robotics or swarm intelligence that are widely researched or areas of interest such as mapping, exploration, foraging, morphogenesis or pattern formation, and search tasks [3]. Autonomous robots finding targets within an unknown environment is a problem that is suitable for a swarm of robots. The area or environment in question can be dangerous or inaccessible to humans or robots could be deployed as secondary operation aiding humans. A swarm robotics approach has some advantages compared to single robots. It is vastly more efficient

${ }^{1}$ Muhammad S. A. Khan is with the Department of Electrical and Electronic Engineering, BRAC University, Dhaka, Bangladesh. E-mail: md.shadnan.azwad.khan@g.bracu.ac.bd

${ }^{2}$ Mohammad S. Hasan is with The School of Computing and Digital Technologies, Staffordshire University, Stoke-on-Trent, UK. E-mail: m.s.hasan@staffs.ac.uk

${ }^{3}$ Tarem Ahmed is with the Department of Computer Science and Engineering, Independent University, Bangladesh (IUB), Dhaka, Bangladesh. E-mail: tarem@iub.edu.bd and robust in its execution due to a parallel autonomous behaviour of the individual in the swarm and the scalable nature of the swarm itself [1]. Sensory information accrued by multiple robots allow for optimization techniques to be used that improve the solution significantly [4]. Given all its advantages, search and rescue solutions using swarm robotics are relatively few leaving a wide possibility for further research. This paper aims to propose a new algorithm for a swarm of robots carrying out search and rescue operations.

This paper is organized into several sections. In section II, past research work and related papers are discussed. Section III introduces and explains the proposed algorithm. Section IV describes the simulation environment and the procedures related to it. In section $\mathrm{V}$, the results of the simulations are used to analyse the proposed algorithm and finally, section VI discusses the performance of the proposed algorithm along with limitations and draws some conclusions and points to some possible future works.

\section{EXISTING WORKS}

Multi-robot systems essentially have three different coordination paradigms: "centralised" architecture where decisionmaking is under control of a single entity, "decentralised hierarchical" architecture where decision-making is based on negotiated through a hierarchy system implemented locally, and "decentralized distributed" architecture where each entity is autonomous and decision-making is completely decentralized according to [5]. Multi-robot system is used in the current state of the art in the field of search and rescue for the reconnaissance and rescuing phases of a search and rescue mission as described in [6] to assist human responders and it has been found that distributed approaches avoid bottlenecks due to overflows in communication links as in centralized approaches.

Particle Swarm Optimization (PSO) first proposed in [7] is an evolutionary computation technique that is inspired by social behaviours of foraging swarms. It is a robust and flexible approach that utilises individual fitness to maximise global performance. It considers present states and best performance in the swarm and past best performances of individuals to move towards an optimised solution towards the goal state. Due to its simplicity and low time and space complexity, PSO is easy to implement and has been adapted for swarm robotics despite being created as a solution to optimisation or estimation problem and has been shown to be an efficient algorithm for many applications [8]. 
There are many examples of search and rescue approaches in which unknown environments are traversed to locate targets at unknown locations such as in [9] where PSO is used with adaptive RSS weighting factor. Another method is shown in [10] with a search algorithm inspired by PSO used to find targets without precise global information where cartesian geometry is used to unify relative coordinate systems to improve robustness and efficiency.

A distributed approach to multi-robot search is proposed in [11] where PSO is modified inspired by chemotaxis behaviour in bacteria. The approach is tested on dynamic environments for the fitness of individuals and for the swarm globally. Local adaptations based on varying neighbourhood sizes are used to test for the change in global fitness achieved through local interactions. The results show that the approach is adaptive in dynamic environments and continue adaptations throughout changes in the environment without loss in performance.

A study in [8] conducts benchmark experiments for multirobot search algorithms inspired by swarm intelligence. Five state-of-the-art algorithms are compared using the nonrealistic simulator MRSim. The performance is measured using the exploration ratio of the environment and its average of 500 iterations. Robotic Darwinian PSO (RDPSO) is shown to have the best performance in the simulated experiments. Moreover, the RDPSO is further compared with two other best-performing algorithms, Aggregations of Foraging Swarm (AFS) and Glowworm Swarm Optimisation (GSO), using a swarm of 14 e-pucks. RDPSO converges to the optimal solution faster and with accuracy GSO closely follows its performance. For RDPSO and GSO, the computational load due to space and time complexities or the communication demands are not significantly higher than the other algorithms.

RDPSO is first proposed in [12] along with Robotic PSO (RPSO) as extensions of Darwinian PSO (DPSO) and PSO, respectively. The techniques are modified for obstacle avoidance and for multi-robot systems. A simulation demonstrates these algorithms performing distributed exploration. The techniques use dynamic topology to split the swarms into sub-swarms over several iterations. There arises a chance of getting stuck in a local minimum that is avoided in the RDPSO but not in the RPSO. RDPSO outperforms RPSO by avoiding local optima using a punish-reward mechanism controlling social exclusion and social inclusion. Therefore, global communication and coordinating system considerations outweigh distance metrics and local minimum when dividing the sub-swarms.

GSO is introduced in [13] as an optimization algorithm that is like but distinct from PSO and Ant Colony Optimisation (ACO). The entities in GSO are thought of as Glowworms that carry a fitness value calculated based on their current location called Luciferin which they broadcast to their neighbours. An individual in the swarm computes its movements based on an adaptive neighbourhood where it probabilistically moves towards a neighbour with a higher luciferin value. The swarm divides into disjoint groups die to these movements based on local information and selective neighbours allowing it to move towards multiple signal sources.

Foraging robots could use path planning in their environment for efficiency. In [14] virtual ants are implemented that use artificial pheromones within a swarm network. This is achieved by local messages forming chains within the robots deposited with artificial pheromones that help with path selection. In [15] and expanded in [16] a novel system architecture with three layers is implemented: human-computer interaction layer (HCI), planning layer and execution layer used for a foraging task in [15] with the swarm using limited local information and no communication other than some central communication with some robots. In [16] a selfassembling and self-reconfiguring robotic swarm with emphasis on organisation structure also uses the architecture.

A search approach using potential field is shown in [17] where a model based upon Coulomb's inverse-square law is used. The system uses positive charges as obstacles are picked up by sensors and, being positive themselves, the robot moves away from obstacles. The target is also positive since it appears as an obstacle to the sensors and while they are still denoted as a positive charge they are identified using a camera. Once a target has been identified the task is said to be completed. Information sharing could be either pessimistic or optimistic where a robot takes information about an obstacle given by another robot and selects a high charge if pessimistic and low if optimistic. The approach is tested on Player/Stage simulator and the sharing systems are found to have similar performance but both outperform systems without sharing.

Probabilistic finite state machines (PFSM) are also used for swarm robotics applications such as in [18] where a foraging swarm is modelled using PFSM with state searching, grabbing, homing, avoidance, deposit and resting. The mathematical aspects are modelled to mimic macroscopic behaviour while geometric methods are used to derive transitional probabilities of the individuals. Player/Stage simulations of the model show promising results for dynamic situations.

\section{THE PROPOSED AlgorithM}

The algorithm utilises a PFSM which offers flexibility in the design of the approach and it also very easy to implement, therefore, it is used for the proposed algorithm. The states for the PFSM may change due to three different reasons:

(i) Time boundaries: a maximum time boundary changes the state taking transitional probabilities into consideration as well.

(i) Transition probabilities: these are generated randomly and updated through social interaction between the swarm due to various scenarios.

(ii) Task events: events pertaining to the swarm task automatically changes the state of the individual robots concerned. 


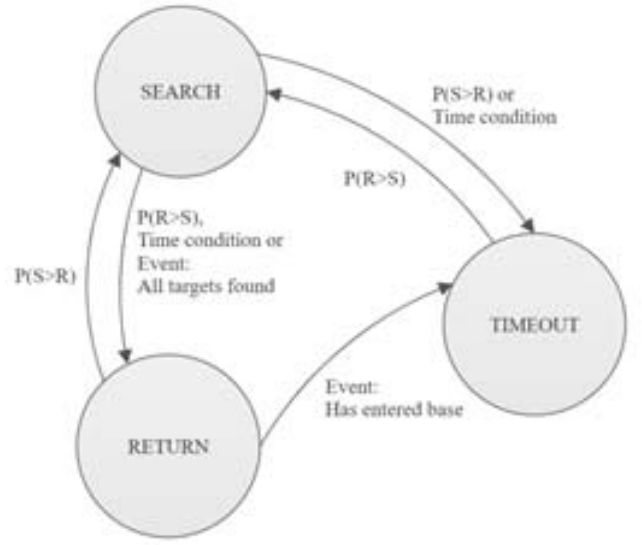

Figure 1: State transition diagram for proposed algorithm

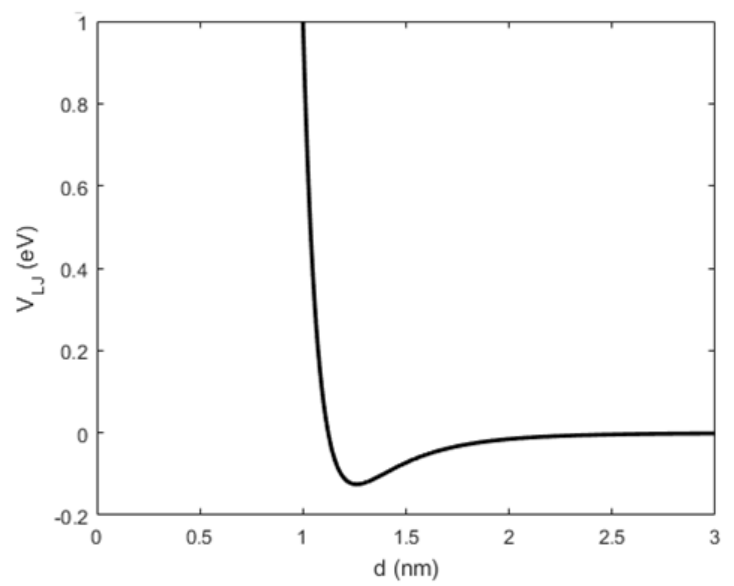

Figure 2: Lennard-Jones potential VLJ of two particles over distance d

The states used for this algorithm are: Timeout, Search, and Return. The general algorithm is shown in Algorithm 1. State Transition Diagram pertaining to the state changes are shown in Figure 1.

\section{A. Timeout}

This state essentially does a timeout for the robot when it is already back in the initial area or base. It does a clean-up of the transition probabilities and after a given time has passed or if other robots have interacted in the meantime allowing for updates in transition probabilities as the robot in timeout to gathers information and interprets them. Depending on probabilities this state transitions from Return and to Search.

\section{B. Search}

The main task of the swarm is to search the environment for target robots. It must not only search its environment it must be done in a distributed way which is also optimised by some method. This method is the use of Lennard-Jones potential and diffusive behaviour. The footbots maintain a target distance of $50 \mathrm{~cm}$.

For this task, the robots in the state of Search work out Lennard-Jones potential its neighbouring search robots and calculates a target distance between itself and its neighbours.

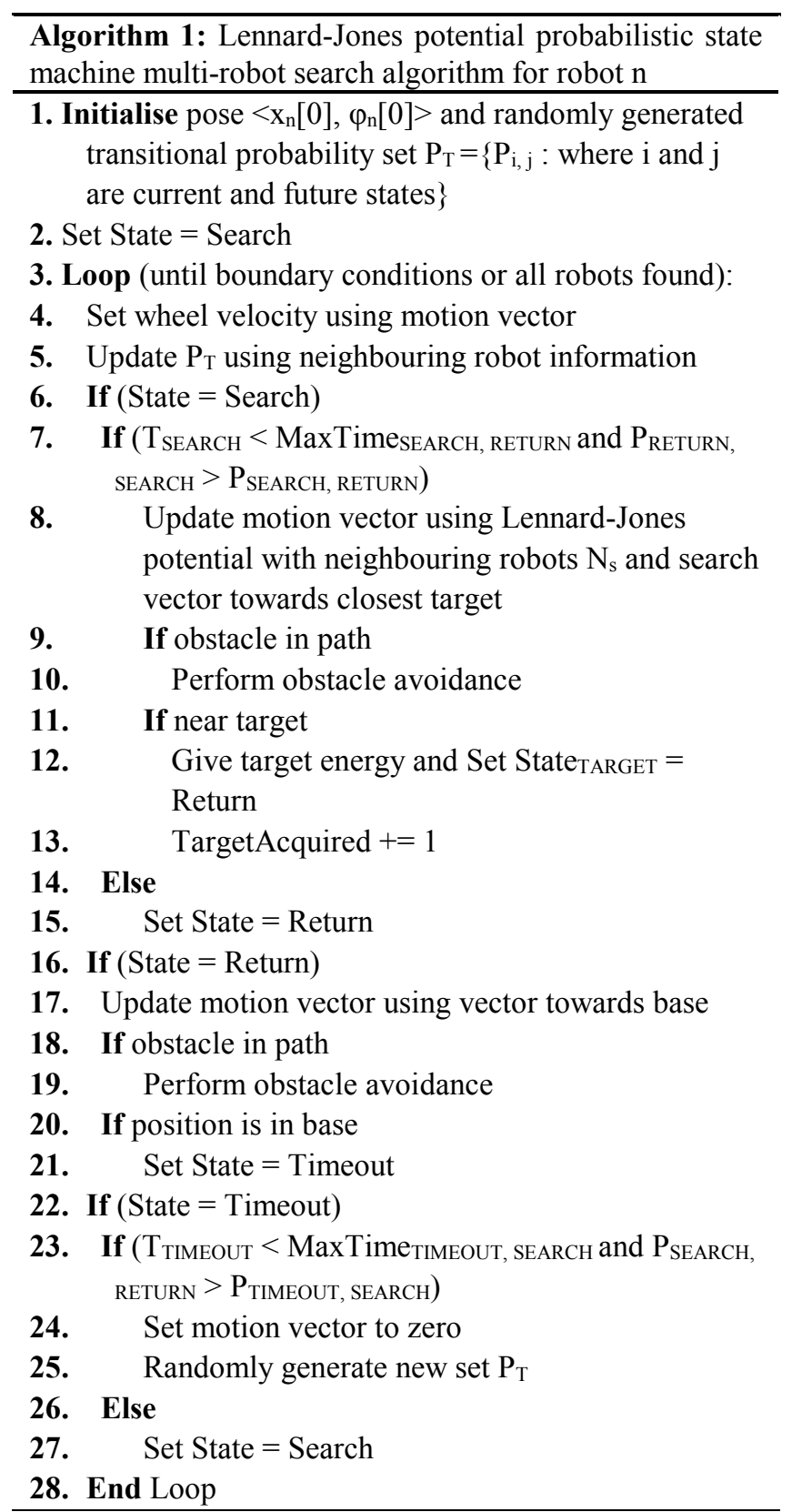

Two-dimensional motion vectors are calculated using a generalised form of the Lennard-Jones potential which is modified for maximum distance between individuals as they search for the targets. The Lennard-Jones potential function is usually expressed as,

$$
V_{L J}=4 \varepsilon\left[\left(\frac{\sigma}{d}\right)^{12}-\left(\frac{\sigma}{d}\right)^{6}\right]
$$

Where the potential well is defined by $\varepsilon$, the inter-particle distance where potential is zero by $\sigma$, the distance between particles by d. Figure 2 shows the graph for Lennard-Jones potential between two charged particles. The targets themselves are given the higher potential. Finding robots in a neighbourhood changes the transitional probabilities of the 
neighbouring swarm robots.

There is also an obstacle avoidance method in place so that robots never come close enough due to the potential function. The targets once found, are given energy to go back to the base. Figure 1 shows the transition of this state while lines 6 to 15 show in further detail the method implemented to search for the targets.

\section{Return}

Allowing the normal rules of time boundaries and transitional probabilities in effect, returning occurs for the whole swarm only when all the targets have been found and returned to the base or the global time boundary is crossed. Upon transition to the state of Return, a robot calculates its vector to the base and adds it to an obstacle avoidance vector given an obstacle appears in its path. It takes the shortest path back considering its environment is dynamic and has other moving robots in it. Obstacle avoidance due to interactions between robot's updates transition probabilities. Upon returning to base the state transitions to Timeout.

\section{SIMULATION AND EXPERIMENTAL SETUP}

\section{A. ARGoS overview}

ARGoS as outlined in [19] is a modular, pluggable, multiphysics engine simulator capable of simulating large heterogeneous swarm robotics in real time with efficiency and flexibility in its design. It can use multiple threads and multiple physics engines and robots can move freely from the simulated space of one engine to another with ease and transparency. Distinctly, ARGoS is implemented in a way that every entity is implemented as a plug-in with easy interface to include custom plug-ins. In simulations, it has been able to simulate up to 10,000 wheeled robots in real-time with full dynamics in place.

\section{B. Swarm task definition}

The goal of the swarm for search and rescue for the simulation is defined as identifying, locating, approaching and returning the targets to initial location of the search and rescue robots. With ending criteria for successful completion being discovery of all target footbots dispersed in the environment.

\section{Robot model and control}

The footbot modelled after the marXbot [20] is already implemented in ARGoS and is used for the simulation for the validation of the proposed approach. The simulation for the proposed algorithm of this paper utilises the following sensors and actuators from the footbot: Differential steering actuator, Omnidirectional camera, Range and bearing sensor, Range and bearing actuator, Ground sensor, Proximity sensor, Light sensor and LED actuator.

The controller used for the footbot comes with predefined structures inherited from ARGoS. The controller is required to specify a control method that is called every time step as well as an initialising method. Methods are also required to reset or destroy data or memory for the controller. Other than that, the controller can have any number of secondary methods to aid the control. For the case of this paper, methods for the PFSM function and the behaviour for each state including motion control and optimisation have been designed.

\section{Simulation setup and Data logging}

The target distance $(75 \mathrm{~cm})$ and the robot diameter $(29 \mathrm{~cm})$ allow for two complete robots in a $1 \mathrm{~m}^{2}$ area as well as a fraction of another robot. This bounds the highest footbot number by the lowest area. In this case, 80 total footbots in $56.25 \mathrm{~m}^{2}$ with a density of 1.42 footbots $/ \mathrm{m}^{2}$. To test for different scenarios and their effects on the proposed approach some variables are selected, these include:

- Different environments designed to test flexibility (for $7.5 \mathrm{~m}$ by $7.5 \mathrm{~m}, 10 \mathrm{~m}$ by $10 \mathrm{~m}, 12.5 \mathrm{~m}$ by $12.5 \mathrm{~m}$, $15 \mathrm{~m}$ by $15 \mathrm{~m}$ and $17.5 \mathrm{~m}$ by $17.5 \mathrm{~m}$ environments and corresponding areas of 56.25, 100, 156.25, 225 and $306.5 \mathrm{~m}^{2}$ )

- Search footbot numbers which are varied to test scalability (tested for 8, 16, 24, 32 and 40 search footbots)

- Target footbot numbers that are varied to test robustness (tested for 8, 16, 24, 32 and 40 target footbots)

The data logged for each scenario using loop functions are: energy of the swarm at each time step, time taken to locate each target, and total target footbots located. The simulation is carried out for each scenario from changing the variables above. The simulation design is as follows:

(i) The environment is fixed, and footbot number is fixed while target numbers are changed

(ii) If environment has not been tested with full range of footbots, the footbot number is changed and simulation started from step (i) again

(iii) If other environments remain to be tested, change to a different environment and start from step (i)

(iv) End simulation and collect data for each separate simulation scenario

Once concluded, the data collected is then used for performance analysis and to test the different factors associated with the variables. The performance is analysed using the following equation where higher values mean better performance,

$$
P(E, t)=\frac{k}{(E)^{0.5}} \times \frac{1}{t}
$$

Where $\mathrm{E}$ is the total energy in Joules expended by swarm after finding the last target, $\mathrm{t}$ is the time taken in seconds to find the last target. Each scenario is analysed using the performance measure. The constant $\mathrm{k}$ is $10^{6} \mathrm{Js}$ scaling the value up and making $\mathrm{P}(\mathrm{E}, \mathrm{t})$ unitless.

\section{RESUltS AND ANALYSIS}

The algorithm is tested on 125 scenarios with performance measures for each. To test flexibility, the environment is kept 


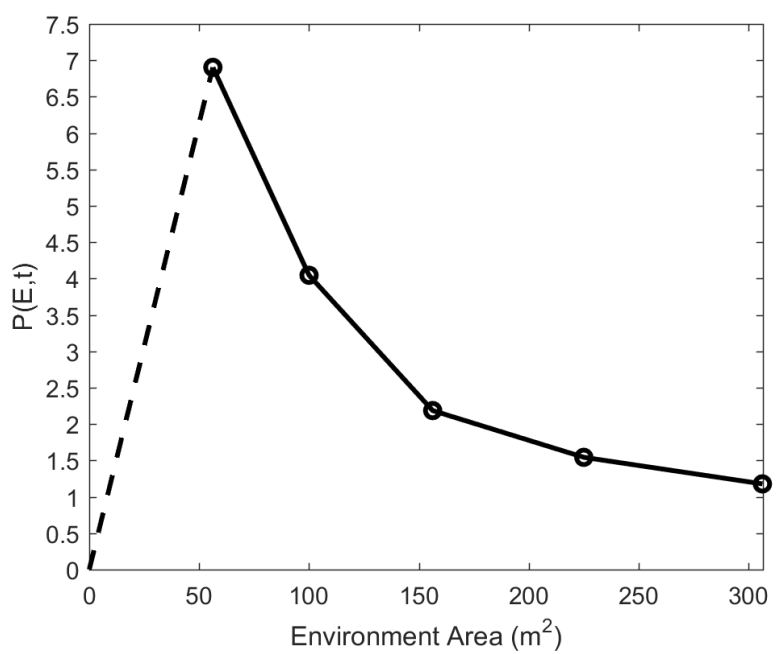

Figure 3: Flexibility test for average performance over environment area

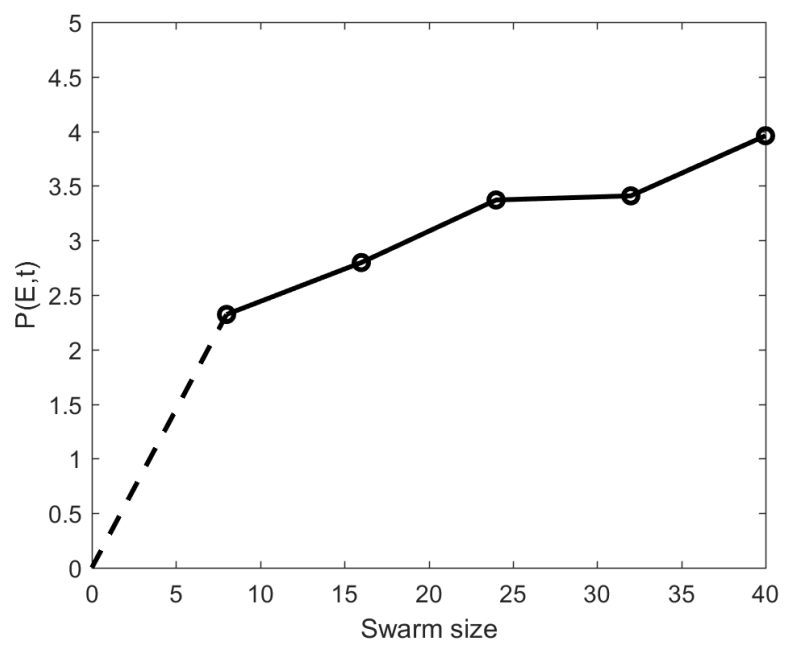

Figure 4: Scalability test for average performance over swarm size

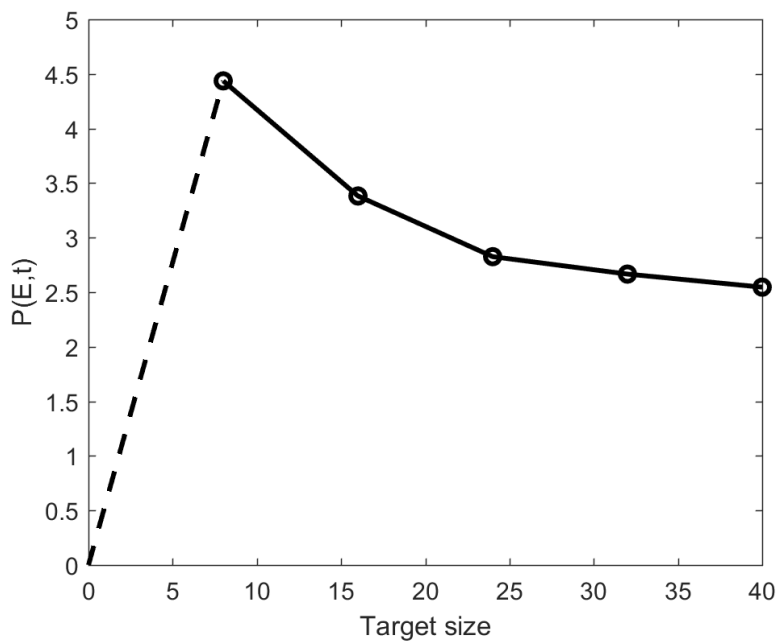

Figure 5: Robustness test for average performance over target size

fixed while search and target robots are varied giving 25 scenarios for each environment. The performance is averaged for each environment, likewise the same is done for
TABLE I. SUMMARY OF MULTI-SEARCH ALGORITHMS IN COMPARISON WITH THE PROPOSED ALGORITHM

\begin{tabular}{|c|c|c|c|}
\hline Aspect & $\begin{array}{c}\text { RDPSO } \\
{[8][12]}\end{array}$ & $\begin{array}{c}\text { GSO } \\
{[8][13]}\end{array}$ & $\begin{array}{l}\text { Proposed } \\
\text { algorithm }\end{array}$ \\
\hline Robot dynamics & $\begin{array}{c}\text { Fractional calcu- } \\
\text { lus }\end{array}$ & - & - \\
\hline $\begin{array}{c}\text { Initial } \\
\text { deployment }\end{array}$ & EST & Random & Random \\
\hline $\begin{array}{c}\text { Obstacle avoid- } \\
\text { ance }\end{array}$ & $\begin{array}{c}\text { Artificial repul- } \\
\text { sion }\end{array}$ & $\begin{array}{c}\text { Low-level } \\
\text { control }\end{array}$ & $\begin{array}{l}\text { Artificial } \\
\text { repulsion }\end{array}$ \\
\hline Communication & $\begin{array}{l}\text { Ad hoc multi- } \\
\text { hop }\end{array}$ & Broadcast & Broadcast \\
\hline $\begin{array}{l}\text { Sub-optima } \\
\text { avoidance }\end{array}$ & $\begin{array}{c}\text { Reward } \\
\text { punishment }\end{array}$ & - & - \\
\hline $\begin{array}{c}\text { Multiple } \\
\text { dynamic sources }\end{array}$ & $\begin{array}{l}\text { Dynamic parti- } \\
\text { tioning and } \\
\text { fuzzy adaptive } \\
\text { behaviour }\end{array}$ & Partitioning & - \\
\hline $\begin{array}{c}\text { Computational } \\
\text { complexity }\end{array}$ & $\mathrm{O}\left(2 \mathrm{~N}_{\mathrm{S}}\right)$ & $\mathrm{O}\left(\mathrm{N}_{\mathrm{S}}\right)$ & $\mathrm{O}\left(\mathrm{N}_{\mathrm{S}}\right)$ \\
\hline $\begin{array}{c}\text { Memory com- } \\
\text { plexity }\end{array}$ & $\mathrm{O}\left(\mathrm{R}_{\mathrm{A}}\right)$ & $\mathrm{O}(1)$ & $\mathrm{O}(1)$ \\
\hline $\begin{array}{l}\text { Communication } \\
\text { complexity }\end{array}$ & $\mathrm{O}\left(\mathrm{N}_{\mathrm{S}}\right)$ & $\mathrm{O}\left(\mathrm{N}_{\mathrm{S}}\right)$ & $\mathrm{O}\left(\mathrm{N}_{\mathrm{S}}\right)$ \\
\hline
\end{tabular}

scalability and robustness test using search and target robots.

Space, time, and communication complexities are also compared to RDPSO and GSO (the best performing algorithms as shown in [8]). Other aspects such as obstacle avoidance and sub-optima avoidance are also compared.

\section{A. Flexibility}

Three different environments are used of areas 56.25, 100, 156.25, 225 and $306.5 \mathrm{~m}^{2}$. The results shown in Figure 3 show that performance is highest for smaller environments. This is anticipated since the communication range and interaction vectors benefit from a shorter range. Smaller portioned groups arise in the larger areas allowing for less computational complexity however overall search time and energy spent in larger environments mean performance decreases. Although the performance, does eventually settle into a constant value. The algorithm is quite flexible because all targets are found eventually although it lacks a proper distribution method for the footbots.

\section{B. Scalability}

Figure 4 shows the average performance for the varying swarm sizes of $8,16,24,32$ and 40 . The best case is for the largest swarm size, with the exception of a slight dip at 32 the overall graph is linear. It has a positive slope that is nearly constant showing the algorithm is definitely scalable and even better when up scaled. The swarm had a difficult time avoiding sub-optima for the swarm size of 32 which might explain its poorer performance.

\section{Robustness}

Varying the target size has a very noticeable and apparent effect, Figure 5 shows a graph which decreases to a constant 
value. The difference between the highest performance and lowest is not great, this is due to the motion vectors depending on targets in some situations to escape sub-optima due to an aspect of the Lennard-Jones potential. The proposed algorithm proves robust for even large target sizes as target size increases from 8, 16, 24, 32 and 40. All the target robots are found and the difference in performance for each increase is within a reasonable range. The value eventually seems to become constant.

\section{Algorithmic complexity and feature comparison}

Further validation of the proposed algorithm can be shown through complexity analysis for some of the leading multisearch algorithms. RDPSO and GSO are compared with the proposed algorithm. Table 1 shows the summary for the comparison. RDPSO has robot dynamics using fractional calculus and sub-optima avoidance while the other two do not have any implement of such kind. The initial deployment of the GSO and proposed algorithm are Random while RDPSO and proposed algorithm based on Lennard-Jones potential utilise artificial repulsion for obstacle avoidance. The communication methods are broadcast for both GSO and proposed while RDPSO uses Ad hoc multi-hop.

However, communication complexity for all three is the same and depends on $\mathrm{N}_{\mathrm{S}}$ which is the neighbouring swarm size. Computation complexity is higher for RDPSO as well as memory complexity since it uses a fractional order series $\mathrm{R}_{\mathrm{A}}$. Memory complexity for GSO and proposed algorithm only depend on fixed number of values taken from previous iteration hence the lower complexity.

\section{CONCLUSION}

The proposed algorithm has shown promise as a proper swarm implementation for Lennard-Jones potential and PFSM for the task of multi-robot search for multiple targets. It is adequately scalable, flexible and robust and shows similar algorithmic complexity to other algorithms of this nature. Due to the nature of PFSM, modifications can be made with ease to allow for higher functionality. It does have issues with sub-optima and, has not been tested extensively. It lacks various features that would be useful such as a way to distribute swarm across environment leading to more flexibility.

Furthermore, it has some trouble with very dynamic environments. There is scope for further work in a real environment with real robots for dynamic multiple targets. Practical testing would reveal more information and remains as future work along with an optimisation technique for the parameters. Cooperation could also be improved between the robots through a different cooperation approach. Further work, therefore, could focus on a multi-robot cooperation and communication framework.

\section{ACKNOWLEDGMENT}

The authors would like to acknowledge an Erasmus+ Inter- national Credit Mobility (ICM) fund for Bangladesh awarded to Dr. Mohammad Hasan at Staffordshire University, UK where Muhammad S. A. Khan was a visiting student when this project began.

\section{REFERENCES}

[1] Y. Tan and Z. Zheng, "Research Advance in Swarm Robotics," Def. Technol., vol. 9, no. 1, pp. 18-39, 2013.

[2] E. Sahin, "Swarm robotics: From sources of inspiration to domains of application," Swarm Robot., pp. 10-20, 2004.

[3] L. Bayindir, "A review of swarm robotics tasks," Neurocomputing, vol. 172, pp. 292-321, 2016.

[4] G. Beni, "From Swarm Intelligence to Swarm Robotics," Swarm Robot., vol. 3342, pp. 1-9, 2005.

[5] R. P. P. Rocha, "Building volumetric maps with cooperative mobile robots and useful information sharing: a distributed control approach based on enthropy," 2006.

[6] M. S. Couceiro, D. Portugal, and R. P. Rocha, "A Collective Robotic Architecture in Search and Rescue Scenarios," Proc. 28th Annu. ACM Symp. Appl. Comput. - SAC '13, pp. 64-69, 2013.

[7] J. Kennedy and R. Eberhart, "Particle swarm optimization," Neural Networks, 1995. Proceedings., IEEE Int. Conf., vol. 4, pp. 1942-1948 vol.4, 1995.

[8] M. S. Couceiro, P. A. Vargas, R. P. Rocha, and N. M. F. Ferreira, "Benchmark of swarm robotics distributed techniques in a search task," Rob. Auton. Syst., vol. 62, no. 2, pp. 200-213, 2014.

[9] K. Derr and M. Manic, "Multi-robot, multi-target particle swarm optimization search in noisy wireless environments," in Proceedings - 2009 2nd Conference on Human System Interactions, HSI '09, 2009, pp. 81-86.

[10] Q. Zhu, A. Liang, and H. Guan, "A PSO-inspired multi-robot search algorithm independent of global information," in IEEE SSCI 2011 - Symposium Series on Computational Intelligence SIS 2011: 2011 IEEE Symposium on Swarm Intelligence, 2011, pp. 74-80.

[11] J. Pugh and A. Martinoli, "Distributed Adaptation in Multi-Robot Search using Particle Swarm Optimization," in Proceedings of the 10th International Conference on the Simulation of Adaptive Behavior, 2008, pp. 393-402.

[12] M. S. Couceiro, R. P. Rocha, and N. M. F. Ferreira, “A novel multi-robot exploration approach based on particle swarm optimization algorithms," Safety, Secur. Rescue Robot. (SSRR), 2011 IEEE Int. Symp., pp. 327-332, 2011.

[13] K. N. Krishnanand and D. Ghose, "Glowworm swarm optimization for simultaneous capture of multiple local optima of multimodal functions," Swarm Intell., vol. 3, no. 2, pp. 87-124, 2009.

[14] A. Campo et al., "Artificial pheromone for path selection by a foraging swarm of robots," Biol. Cybern., vol. 103, no. 5, pp. 339-352, 2010.

[15] Y. Leng, Y. Zhang, X. He, and W. Zhou, "Algorithm for foraging and building task on a novel swarm robotic platform," Robot. Biomimetics 2014 IEEE Int. Conf., 2014.

[16] Y. Leng, Y. Zhang, W. Zhang, X. He, D. Bian, and W. Zhou, "SociBuilder: A novel task-oriented swarm robotic system," Robot. Biomimetics 2015 IEEE Int. Conf., pp. 48-53, 2015.

[17] J. L. Baxter, E. K. Burke, J. M. Garibaldi, and M. Norman, "Multi-robot search and rescue: A potential field based approach," in Autonomous Robots and Agents, vol. 76, 2007, pp. 9-16.

[18] W. Liu, A. F. T. Winfield, and J. Sa, "Modelling Swarm Robotic Systems: A Case Study in Collective Foraging," Proceeding Towar. Auton. Robot. Syst., pp. 25-31, 2002.

[19] C. Pinciroli et al., "ARGoS: A modular, parallel, multi-engine simulator for multi-robot systems," Swarm Intell., vol. 6, no. 4, pp. 271-295, 2012.

[20] M. Bonani et al., "The marXbot, a miniature mobile robot opening new perspectives for the collective-robotic research," in IEEE/RSJ 2010 International Conference on Intelligent Robots and Systems, IROS 2010 - Conference Proceedings, 2010, pp. 4187-4193. 